\title{
Non-metabolisable insulin glargine does not promote breast cancer growth in a mouse model of type 2 diabetes
}

\author{
Emily J. Gallagher ${ }^{1}$ - Zara Zelenko ${ }^{1}$ Aviva Tobin-Hess ${ }^{1}$ - Ulrich Werner ${ }^{2}$. \\ Norbert Tennagels $^{2} \cdot$ Derek LeRoith $^{1}$
}

Received: 15 April 2016/Accepted: 3 May 2016 / Published online: 30 May 2016

(C) Springer-Verlag Berlin Heidelberg 2016

\begin{abstract}
Aims/hypothesis Previous epidemiological studies have reported a potential link between insulin analogues and breast cancer; however, a prospective randomised controlled trial showed neutral effects of insulin glargine on cancer risk. Insulin glargine is metabolised in vivo to an M1 metabolite. A question remains whether a subset of individuals with slower rates of glargine metabolism or who are on high doses could, theoretically, have an increased risk of cancer progression if a tumour is already present. In this study, we aimed to determine whether a non-metabolisable form of insulin glargine induced murine breast cancer growth.

Methods A mouse model of type 2 diabetes (MKR) was used for these studies. MKR mice were injected with two murine mammary cancer cell lines: Mvt-1 cells (derived from MMTV-c-Myc/Vegf tumours) and Met1 cells (derived from MMTV-polyoma virus middle T antigen tumours). Mice were treated with $25 \mathrm{U} / \mathrm{kg}$ per day of the long-acting insulin analogues, insulin glargine, insulin detemir, insulin degludec or non-metabolisable glargine, or vehicle.
\end{abstract}

Electronic supplementary material The online version of this article (doi:10.1007/s00125-016-4000-x) contains peer-reviewed but unedited supplementary material, which is available to authorised users.

Emily J. Gallagher

emily.gallagher@mssm.edu

1 Division of Endocrinology, Diabetes and Bone Diseases, Department of Medicine, Icahn School of Medicine at Mount Sinai, One Gustave L Levy Place, Box 1055, New York, NY 10029, USA

2 Diabetes Research \& Translational Medicine, Insulin Biology, Sanofi-Aventis Deutschland GmbH, Frankfurt am Main, Germany
Results No difference in tumour growth was seen in terms of tumour size after insulin glargine, detemir, degludec or vehicle injections. Non-metabolisable glargine did not increase tumour growth compared with insulin glargine or vehicle. Insulin glargine and non-metabolisable glargine led to insulin receptor phosphorylation in vivo rather than IGF-1 receptor phosphorylation.

Conclusions/interpretation These results demonstrate that in a mouse model of type 2 diabetes, at high concentrations, basal insulin analogues and a non-metabolisable glargine analogue do not promote the progression of breast tumours.

Keywords Breast cancer · Insulin analogues · Insulin-like growth factor receptor $\cdot$ Insulin receptor · Type 2 diabetes
Abbreviations
Dn Dominant negative
$\mathrm{FVB} / \mathrm{n} \quad$ Friend virus B/NIH
IGF-1R Insulin-like growth factor 1 receptor
IR Insulin receptor
IR-A Insulin receptor isoform A
IR-B Insulin receptor isoform B
LC/MS Liquid chromatography-tandem mass spectrometry
Met1 MMTV-polyoma virus middle T antigen derived breast cancer cell line
MKR Muscle (M)-IGF-1R-lysine (K)-arginine (R) genetically modified mice
Mvt-1 MMTV-c- $M y c / V e g f$ derived breast cancer cell line
rhIGF-1 Recombinant human insulin-like growth factor 1 


\section{Introduction}

Over $25 \%$ of adult patients with diabetes in the USA take insulin therapy [1]. Questions have been raised regarding the potential for certain insulin analogues to have growthpromoting effects in individuals with cancer. For insulin glargine, this association was initially reported in a retrospective study published by Hemkens et al in 2009 [2]. However, a subsequent prospective clinical trial, the Outcome Reduction with Initial Glargine Intervention (ORIGIN) trial, with 12,500 patients found no increased cancer incidence in insulin glargine users [3]. However, although methodological limitations were apparent in the study by Hemkens et al, and other related retrospective studies [4, 5], a debate has continued on whether insulin glargine could promote tumour progression in individuals with established tumours [6].

The postulated mitogenic potential of insulin glargine is based on its greater affinity for IGF-1 receptor (IGF-1R) than human insulin in vitro, and this finding is comparable with that for AspB10 insulin, which is the only insulin analogue with proven tumourigenicity in animals. However, when glargine is injected into individuals with type 2 diabetes it is rapidly metabolised into intermediate metabolites, the M1 and M2 metabolites. The M1 metabolite accounts for $>90 \%$ of plasma insulin content after administration of insulin glargine to animals and patients, whereas M2 metabolite is usually undetectable, and parent glargine accounts for $<10 \%$ of plasma insulin [7, 8]. In vitro, the M1 metabolite has an affinity for IGF-1R similar to that of human insulin, and has equivalent mitogenic potency to that of human insulin [9]. However, some variability has been observed in the inter-individual rates of metabolism of insulin glargine into the M1 metabolite [7], raising questions about whether in some individuals on chronic high doses of insulin glargine, sufficient circulating concentrations of the parent compound could be reached that could lead to in vivo IGF-1R activation, and potentially lead to increased growth of tumours expressing IGF-1R. We modelled this scenario by examining the effect of a nonmetabolisable insulin glargine analogue (A21Gly,DiD-Arg) on the growth of murine breast cancers in a mouse model of type 2 diabetes, the MKR mouse.

\section{Methods}

Insulin analogues Insulin analogues were mainly produced by Sanofi R\&D Diabetes Division, Frankfurt, Germany. Recombinant human insulin (Insuman Rapid), AspB10 (X-10) insulin, insulin degludec, insulin glargine and A21Gly,DiD-Arg insulin were produced by recombinant DNA techniques or enzymatic semi-synthesis, purified for homogeneity and made available by Process Development Biotechnology (Sanofi, Frankfurt, Germany). Insulin detemir
(Levemir) was produced by Novo Nordisk, Bagsværd, Denmark, and obtained commercially. Recombinant human IGF-1 (rhIGF-1) was obtained from Ipsen (Ipsen, Brisbane, CA, USA). The amino acid sequences of the insulin analogues compared with human insulin are shown in Fig. 1. AspB10 insulin has an amino acid substitution of histidine at position 10 on the B chain for aspartic acid. Insulin degludec was generated by the addition of a fatty acid (hexadecanedioic acid) by a $\gamma$-L-glutamic acid linker acylated to lysine at B29 of a truncated form of insulin, lacking the C-terminal threonine of the $\mathrm{B}$ chain [10]. Insulin glargine has two L-arginine residues added to the $\mathrm{C}$-terminal of the $\mathrm{B}$ chain and an amino acid substitution of glycine (instead of asparagine) at position 21 of the A chain [11]. Non-metabolisable insulin glargine (A21Gly,DiD-Arg) has the same amino acid sequence as insulin glargine but with D-arginine residues at B31 and B32 instead of L-arginine residues [8]. Insulin detemir is also a truncated form of human insulin, lacking threonine at position 30 of the $\mathrm{B}$ chain, and with the addition of a tetradecanoic (myristic) acid, acylated to lysine at B29 [12]. Prior to injection into the mice, insulin analogues (human insulin, AspB10 insulin, insulin degludec, insulin detemir) or rhIGF-1 were diluted in PBS or in a vehicle solution containing water, glycerol and adjusted to $\mathrm{pH} 4$ with hydrochloric acid and $\mathrm{NaOH}$ (insulin glargine or A21Gly,DiD-Arg).

Animal studies All animal study protocols were approved by the Icahn School of Medicine Institutional Animal Care and Use Committee. We used a previously described mouse model of type 2 diabetes for these studies, the MKR mouse [13, 14]. Briefly, muscle (M)-IGF-1R-lysine (K)-arginine (R) mice (MKR) transgenically express a human dominant negative (dn)IGF-1R with a lysine to arginine point mutation in the ATP binding site in the tyrosine kinase domain [13]. The dnIGF-1R is expressed in skeletal muscle under control of the muscle creatine kinase promoter, leading to skeletal muscle insulin resistance, with subsequent whole body insulin resistance [13].

All mice used in these studies were female, on an $\mathrm{FVB} / \mathrm{n}$ (Friend virus B/NIH) background and were between $8-12$ weeks of age when tumour cells were injected. Mice were bred and housed in the Icahn School of Medicine at Mount Sinai Center for Comparative Medicine and Surgery. Mice were maintained on a $12 \mathrm{~h}$ light/dark cycle with free access to water and standard mouse chow (Picolab Rodent Diet no. 5053; LabDiet, St Louis, MO, USA).

Orthotopic tumour models and insulin treatment The Mvt-1 tumour cell line was derived from MMTV-c-Myc/ Vegf explant cultures [15]; the Metl tumour cell line was derived from the MMTV-polyoma virus middle T antigen explant cultures as previously described [16]. Mvt-1 and Met1 cells were cultured in Dulbecco's Modified Eagles Medium 


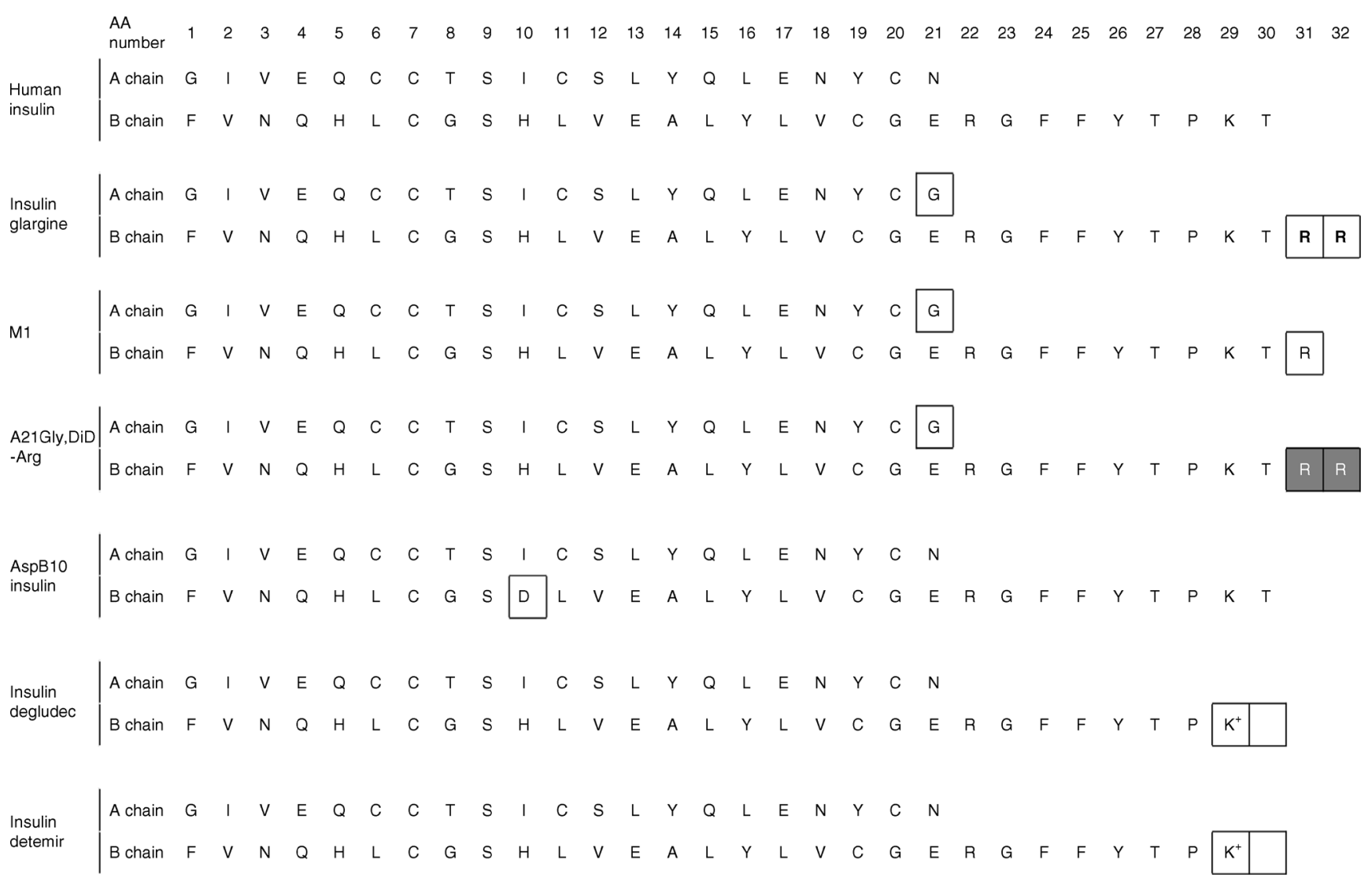

Fig. 1 Amino acid sequences of human insulin and insulin analogues. Human insulin comprises an A chain with 21 amino acids (AAs) and a B chain with 30 AAs. A box around the AA letter indicates that the AA is altered, removed or added in the analogue compared with human insulin. Insulin glargine is metabolised to the M1 metabolite by removal of one Cterminal arginine on the B chain. A21Gly,DiD-Arg has the same AA sequence as insulin glargine, but the $\mathrm{C}$-terminal arginines of parent insulin glargine are L-isomers (highlighted in bold font), and are D-isomers in non-metabolisable glargine (highlighted in grey). AspB10 insulin has an AA substitution of aspartic acid in place of histidine at position 10 on the $\mathrm{B}$ chain. $\mathrm{K}^{+}$at position 29 on the $\mathrm{B}$ chain of insulin degludec and insulin detemir indicates the addition of a fatty acid: hexadecanedioic acid (degludec) or tetradecanoic acid (detemir)
(DMEM), supplemented with 10\% FBS (Invitrogen, Grand Island, NY, USA), with $100 \mathrm{U} / \mathrm{ml}$ penicillin and $100 \mu \mathrm{g} / \mathrm{ml}$ streptomycin (Mediatech, Manassas, VA, USA) at $37^{\circ} \mathrm{C}$ in $5 \%$ $\mathrm{CO}_{2}$ with $95 \%$ humidity. Preparation and tumour cell injection was performed as described previously [14, 17]. A total of 100,000 Mvt-1 cells or 250,000 Metl cells were inoculated into the fourth mammary fat pad of 8-12 week old female virgin MKR mice. Tumours were allowed to grow until they were $2-3 \mathrm{~mm}$ in diameter. At that point, mice were divided into treatment groups matched for tumour size. Mice were treated with rhIGF-1 $1 \mathrm{mg} / \mathrm{kg}$ twice daily by i.p. injection, and AspB10 insulin, recombinant human insulin, insulin glargine, A21Gly,DiD-Arg insulin, insulin degludec, insulin detemir or vehicle $12.5 \mathrm{U} / \mathrm{kg}$ twice daily by s.c. injection [18]. Before and during treatment, tumour growth was measured in three dimensions using callipers. Tumour volume was calculated by: $4 / 3 \times \pi \times r_{1} \times r_{2} \times r_{3}$ ( $r=$ radius $)$. At the end of the study, mice were euthanised, and tumours were removed and weighed. Tumours were flash frozen in liquid nitrogen for subsequent protein isolation and analysis.
Insulin tolerance test Female wild-type $\mathrm{FVB} / \mathrm{n}$ mice were deprived of food, but had free access to water for $2 \mathrm{~h}$ prior to initiation of the study. Baseline blood glucose was measured from tail vein whole blood using a glucometer (Bayer Contour Next, Bayer Healthcare, Mishawaka, IN, USA) immediately before injection (time 0 ). Mice were injected with $1 \mathrm{U} / \mathrm{kg}$ of human insulin, insulin glargine, non-metabolisable insulin glargine or an equivalent volume of vehicle (PBS) by subcutaneous injection. Glucose was measured from the tail vein $15,30,45$ and 60 min after injection, at which time the mice were refed.

Receptor binding assays The binding of insulin, insulin glargine, glargine M1 metabolite, A21Gly,DiD-Arg and IGF-1 to insulin receptor isoform A (IR-A) and IR-B were analysed using a competitive binding assay, using methods as previously described [8, 9], except that for these assays the plasma membranes were enriched from Chinese hamster ovary cells overexpressing either human IR-A or IR-B, and 
competitive binding assays were performed using $110 \mathrm{pmol}$ A $14\left[{ }^{125} \mathrm{I}\right]$-insulin.

Insulin glargine metabolite measurements MKR mice were treated for 1 week with insulin glargine or non-metabolisable insulin glargine (12.5 U/kg twice daily s.c.). A terminal bleed was performed an hour after the last injection. Plasma concentrations of insulin glargine and its M1 and M2 metabolites were determined by liquid chromatography-tandem mass spectrometry (LC/MS) using methods previously described [19].

Protein extraction and analysis Protein extraction and western blot analysis was performed as previously described [14, 20]. The following antibodies were used: anti-phospho IGF$1 \mathrm{R} \beta^{(\mathrm{Tyr} 1135 / 1136)} /$ Insulin Receptor $\beta^{(\text {Tyr1150/1151) }}$, anti-total Insulin Receptor $\beta$ and total IGF-1R $\beta$ (Cell Signaling Technology, Danvers, MA, USA) and anti- $\beta$-actin (Sigma Aldrich, St Louis, MO, USA) at concentrations detailed in electronic supplementary material (ESM) Table 1. Western blots were imaged and quantified by densitometry after incubation with LI-COR IRdye secondary antibodies with a LICOR scanner and LI-COR Image Studio software (LI-COR, Lincoln, NE, USA).

\section{In vitro studies}

The methods for the in vitro studies, the results of which are presented in ESM Figs 1 and 2, are detailed in ESM Methods.

Statistical analysis One-way ANOVA with Tukey's HSD post hoc test was performed to determine statistically significant differences between the means of multiple groups. Statistical analysis was performed using the statistics software package SPSS Statistics (IBM, Armonk, NY, USA). Data are presented as mean $\pm \mathrm{SEM}$.

\section{Results}

No increase in primary Mvt-1 or Met1 tumour growth was observed after treatment with insulin glargine, insulin detemir or insulin degludec We first compared the in vivo effects of the long-acting basal insulin analogues insulin glargine, insulin detemir and insulin degludec on breast cancer growth, compared with AspB10 insulin and rhIGF-1 that we have previously found to increase tumour growth in these tumour models [18]. Mice were injected with Mvt-1 or Met1 cells and divided into treatment groups, as described in the Methods section. Next, $12.5 \mathrm{U} / \mathrm{kg}$ twice daily of insulin glargine, insulin detemir, insulin degludec, AspB10 insulin, or $1 \mathrm{mg} / \mathrm{kg}$ twice daily $\mathrm{rhIGF}-1$ or vehicle were administered for 2 weeks ( $n=12$ per group). After 2 weeks, mice treated with AspB10 insulin had larger Mvt-1 tumour volume $\left(260 \pm 17.6 \mathrm{~mm}^{3}\right)$ than vehicle-treated mice $\left(176.8 \pm 17.1 \mathrm{~mm}^{3} ; p<0.05\right)$. Similarly, rhIGF-1-treated mice had larger tumours $\left(269.9 \pm 21.7 \mathrm{~mm}^{3}\right)$ than vehicle-treated mice $(p<0.01)$. Treatment with insulin glargine, insulin detemir or insulin degludec did not increase Mvt-1 tumour growth compared with vehicle treatment (Fig. 2a, b). It has been suggested that the in vitro mitogenic effects of insulin analogues may be related to a higher relative expression of IGF-1R than insulin receptor (IR) in cancer cell lines [21]. We examined the relative expression of IGF-1R and IR in Mvt-1 and Met1 cells compared with that in human MCF7 and MDA-MB-231 cell lines by western blot. Consistent with previous studies, we found that MCF7 cells had a higher IGF1R:IR ratio on western blot, while the other cell lines had a lower IGF-1R:IR ratio, although both Mvt-1 and Met1 cell lines expressed both receptors (ESM Fig. 1). Despite the relatively lower IGF-1R:IR ratio, Mvt-1 and Met1 tumours grow larger in response to IGF-1 and AspB10 insulin in the MKR mice [18]. Three separate tumour studies were performed in the MKR mice, using insulin glargine, insulin detemir and insulin degludec in both Mvt-1 and Met1 tumours. None of the studies found an increase in Mvt-1 or Met1 tumour growth in mice treated with these analogues over vehicle.

Chronic administration of insulin glargine led to phosphorylation of IR in tumours in the MKR mice Previous in vitro studies have reported that insulin glargine has greater affinity for IGF-1R than human insulin, and may mediate mitogenic effects in tumours that express IGF-1R. After 2 weeks of treatment, mice were dissected $1 \mathrm{~h}$ after the last treatment injection. Consistent with our previous studies [18], chronic rhIGF-1 administration led to IGF-1R and IR phosphorylation or IGF-1R/IR hybrid receptor phosphorylation in the tumours, visible on the western blot as receptor phosphorylation at both $95 \mathrm{kDa}$ and $97 \mathrm{kDa}$ (Fig. 3a). ESM Fig. 2 demonstrates the different molecular mass of IR $\beta$ and IGF$1 \mathrm{R} \beta$ phosphorylation on western blot. AspB10 insulin, insulin glargine, insulin detemir and insulin degludec led to IR phosphorylation in the tumours, visible at $95 \mathrm{kDa}$ on the western blot, but not IGF-1R phosphorylation (Fig. 3a). Greater IR phosphorylation was observed in tumours from the mice treated with AspB10 insulin (Fig. 3b) than the other insulin analogues. This finding is consistent with previous studies that report the mitogenic effects of AspB10 insulin are mediated by sustained IR phosphorylation [22].

Treatment with non-metabolisable insulin glargine did not increase Mvt-1 or Met1 tumour growth compared with parent insulin glargine or vehicle As described in the Methods section, a non-metabolisable form of insulin glargine was generated by replacing the L-arginine residues at position 31 and 32 on the B chain of insulin with D-arginine residues 

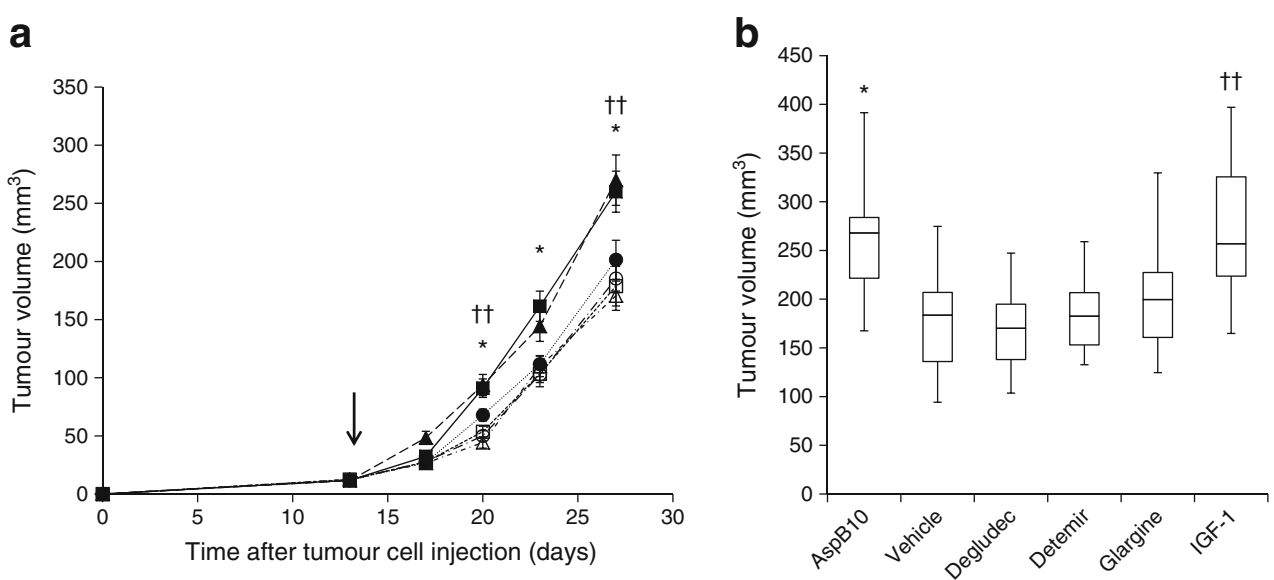

Fig. 2 Long-acting insulin analogues did not increase breast cancer growth over vehicle. (a) Mvt-1 tumour growth with mean tumour volumes in each group ( $n=12$ per group). Mvt-1 cells were injected at day 0 , insulin injections began as indicated by the vertical arrow. Black squares, AspB10; white squares, vehicle; white triangles, degludec; white circles, detemir; black circles, glargine; black triangles, IGF-1. Error bars

(A21Gly,DiD-Arg) [8]. These D-arginines are not cleaved by carboxypeptidases. The in vitro binding affinity of insulin glargine, the M1 glargine metabolite, non-metabolisable A12Gly,DiD-Arg insulin and IGF-1 to IR-A and IR-B are presented in Table 1. The affinity of non-metabolisable glargine, glargine and the glargine M1 metabolite for IR-A and IR-B were similar to each other, while as previously published, binding to IGF-1R was similar between glargine and non-metabolisable glargine, but the glargine M1 metabolite had a lower IGF-1R binding affinity (Table 1).

To examine the in vivo biological activity of nonmetabolisable insulin glargine on glucose lowering, compared with parent insulin glargine, an insulin tolerance test was performed in wild-type FVB/n mice (Fig. 4a) using human insulin, insulin glargine, non-metabolisable glargine $(1 \mathrm{U} / \mathrm{kg})$ and PBS. At $60 \mathrm{~min}$, glucose levels declined to $49.0 \pm 9.4 \%$ of baseline in the human insulin-treated group, $38.1 \pm 7.7 \%$ in the glarginetreated group and $33.1 \pm 3.1 \%$ in the group treated with nonmetabolisable glargine, and rose to $115.5 \pm 2.8 \%$ in the PBStreated group, with no difference between the three insulin analogue groups at any time point; however, in all groups, glucose levels were significantly lower than the level in the PBS group (Fig. 4a). To determine the concentrations of parent glargine and its M1 metabolite, MKR mice were injected with insulin glargine or non-metabolisable glargine (12.5 U/kg twice daily) for 1 week and the plasma levels of glargine parent compound and glargine metabolites M1 and M2 were measured by LC/MS $60 \mathrm{~min}$ after the last injection. In the glargine-treated group, no detectable glargine was found in the plasma, but the M1 metabolite was detected $(1140.1 \pm 21.9 \mathrm{pmol} / \mathrm{l})$, while in the nonmetabolisable glargine-treated group, glargine was detected (1637.5 \pm 20.6 pmol/l), but no M1 metabolite (Fig. 4b).

represent SEM. (b) Mvt-1 tumour volume at the end of the study (day $27)$ demonstrated as a box and whisker plot ( $n=12$ per group). The horizontal line is the median for each group, the boxes represent the first and third quartiles, and the whiskers represent the range of the data. ${ }^{*} p<0.05$ for the AspB10 group vs vehicle group. ${ }^{\dagger \dagger} p<0.01$ for the rhIGF-1 group vs vehicle group

To determine if this non-metabolisable insulin glargine would lead to mitogenic effects in vivo, MKR mice were injected with Mvt-1 and Met1 cells and treated with vehicle, insulin glargine or non-metabolisable glargine for 2 weeks. No difference in the growth of Mvt-1 or Met1 tumours was found between MKR mice treated with vehicle, insulin glargine or non-metabolisable glargine (Fig. 5a, ESM Fig. 3). These experiments were both repeated once and compared with the findings for human insulin and AspB10 insulin. No increase in Mvt-1 tumour growth was found with either analogue above that with human insulin (Fig. 5b). Western blot analysis revealed that insulin glargine and non-metabolisable glargine both led to IR phosphorylation in Mvt-1 tumours, rather than IGF-1R phosphorylation, as demonstrated by phosphorylation of the $95 \mathrm{kDa}$ band on the western blot (Fig. 5c). No difference in IR phosphorylation was observed between human insulin, insulin glargine or non-metabolisable glargine (Fig. 5d).

\section{Discussion}

Our results demonstrate that in this tumour model none of the long-acting basal insulin analogues in current clinical use promote the growth of Mvt-1 or Met1 tumours in the MKR mouse, when compared with human insulin or vehicle treatment. Furthermore, all of these analogues led to phosphorylation of IR in vivo, rather than IGF-1R phosphorylation, which only occurred with rhIGF-1. This is the first in vivo study examining the effects of a non-metabolisable form of insulin glargine on tumour progression and receptor phosphorylation. We found that in vivo, non-metabolisable glargine (A21Gly, DiD-Arg) did not promote tumour growth over parent glargine, vehicle or human insulin. In addition, treating 
a

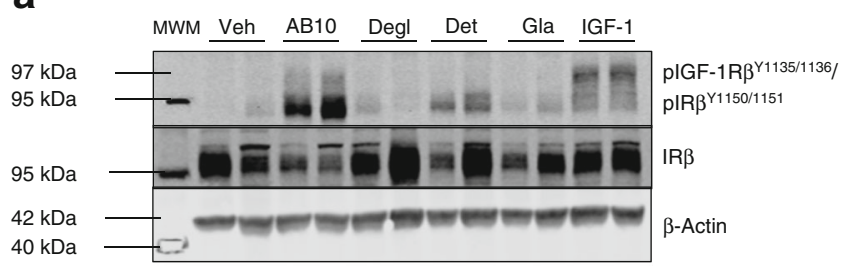

b

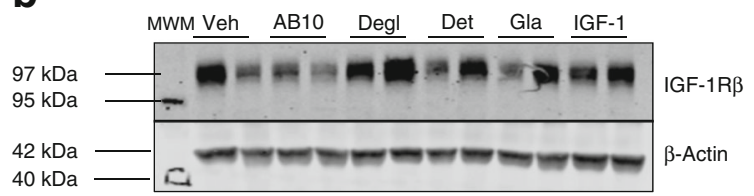

C

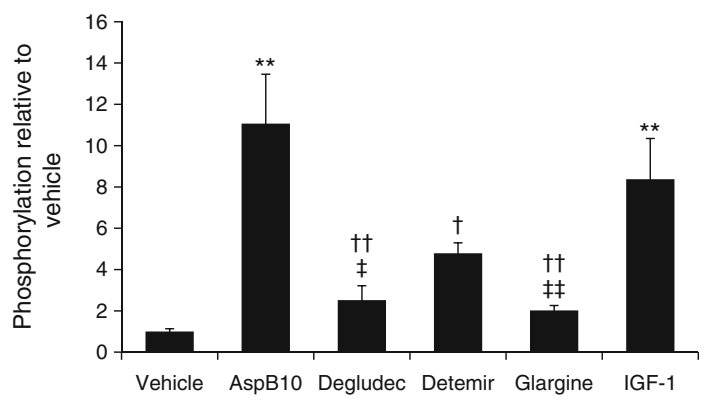

Fig. 3 Insulin degludec, detemir and glargine led to IR phosphorylation in breast tumours. Representative cropped western blot of Mvt-1 tumour lysates from MKR mice treated with vehicle (Veh), $12.5 \mathrm{U} / \mathrm{kg}$ twice daily of AspB10 (AB10), insulin degludec (Degl), insulin detemir (Det), insulin glargine (Gla) and $1 \mathrm{mg} / \mathrm{kg}$ twice daily rhIGF-1 (IGF-1) (a, b). Western blot analysis revealed that the insulin analogues led to IR phosphorylation; rhIGF-1 led to IGF-1R and IR or IGF-1R/IR hybrid receptor phosphorylation. MWM indicates the molecular mass marker lane (a, b). (c) Densitometric analysis of western blot receptor phosphorylation to total IR $\beta$ expression in the tumours, expressed relative to vehicle-treated mice, $n=12$ per group. Bars represent the mean for each group, and error bars are SEM. ${ }^{* *} p<0.01$ vs Vehicle; ${ }^{\dagger} p<0.05,{ }^{\dagger \dagger} p<0.01$ vs AspB10; ${ }^{\star} p<0.05,{ }^{\star \ddagger} p<0.01$ vs rhIGF-1

animals with this non-metabolisable form of insulin glargine did not lead to activation of IGF-1R in the tumours. Overall, these findings are reassuring, given the large number of a

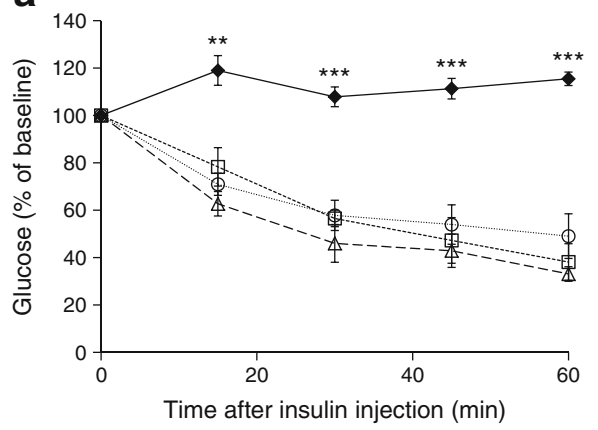

b

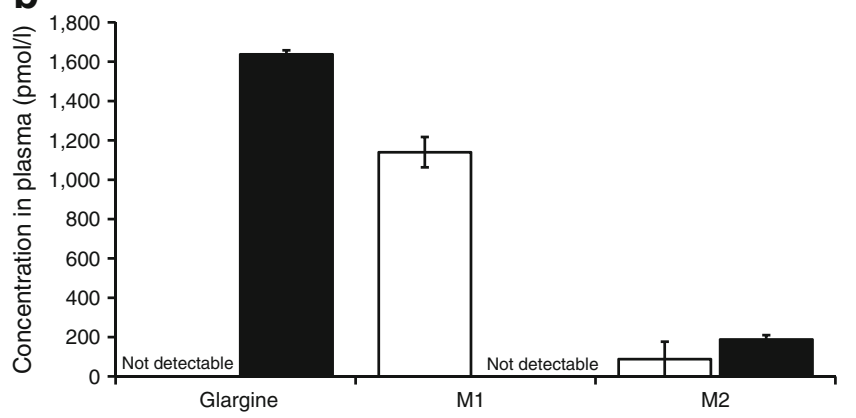

Fig. 4 The metabolic effects and metabolism of insulin glargine and nonmetabolisable insulin glargine in vivo. (a) An insulin tolerance test was performed in wild-type mice using $1 \mathrm{U} / \mathrm{kg}$ of the insulin analogues: human insulin (white circles), parent insulin glargine (white squares) and A21Gly,DiD-Arg (white triangles) and PBS (black diamonds); ( $n=3-4$ per group). ${ }^{* *} p<0.01$ and ${ }^{* * *} p<0.001$ between the PBS group and all other groups. (b) The metabolites of insulin glargine and A21Gly,DiD-Arg in MKR mice after 1 week of treatment with glargine (white bars) or A21Gly,DiD-Arg (black bars) $12.5 \mathrm{U} / \mathrm{kg}$ twice daily ( $n=2$ per group). Graphs represent the mean of each group and error bars represent SEM

patients with breast cancer who have type 2 diabetes, and the high proportion of patients with type 2 diabetes who are treated with basal insulin analogues.

A previous limiting factor in the study of insulin analogues in animal models is the high mortality rates of animals receiving high doses of insulin [23]. Owing to the insulin resistance in the metabolic tissue of MKR mice, a total daily dose of $25 \mathrm{U} / \mathrm{kg}$ was administered in these studies (in two divided
Table 1 Insulin analogue receptor binding affinity

\begin{tabular}{lccc}
\hline Analogue & $\begin{array}{l}\text { IR-A affinity IC50 } \\
\text { (nmol/l) }\end{array}$ & $\begin{array}{l}\text { IR-B affinity IC50 } \\
\text { (nmol/l) }\end{array}$ & $\begin{array}{l}\text { IGF-1R affinity IC50 } \\
\text { (nmol/l) (Ref [8] })\end{array}$ \\
\hline Human insulin & $0.87 \pm 0.13$ & $0.84 \pm 0.24$ & $375.0 \pm 61.9$ \\
Glargine & $1.37 \pm 0.16$ & $1.38 \pm 0.29$ & $20.3 \pm 2.4$ \\
Glargine M1 & $1.33 \pm 0.19$ & $1.73 \pm 0.05$ & $645 \pm 21.7$ \\
A21Gly,DiD-Arg insulin & $1.83 \pm 0.19$ & $1.54 \pm 0.76$ & $22.8 \pm 2.7$ \\
IGF-1 & $61.26 \pm 9.40$ & $509.53 \pm 6.02$ & $0.68 \pm 0.17$ \\
\hline
\end{tabular}

Data are presented as mean \pm SEM

IR-A, IR-B and IGF-1R binding affinity of insulin, glargine, glargine M1 metabolite and non-metabolisable glargine (A21Gly,DiD-Arg)

IR-A and IR-B data are representative of two independent experiments with $n=4$ per group. IGF-1R studies are presented for comparison and were previously performed and published in reference [8], as indicated 


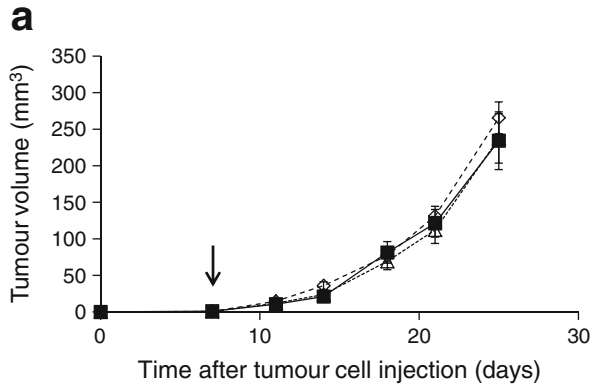

C

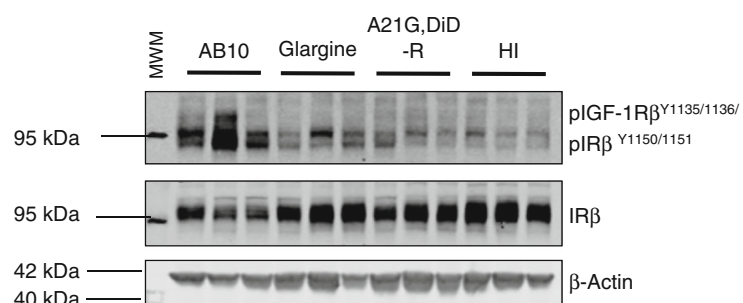

b

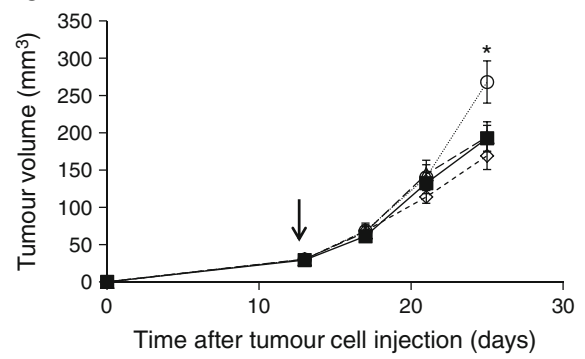

d

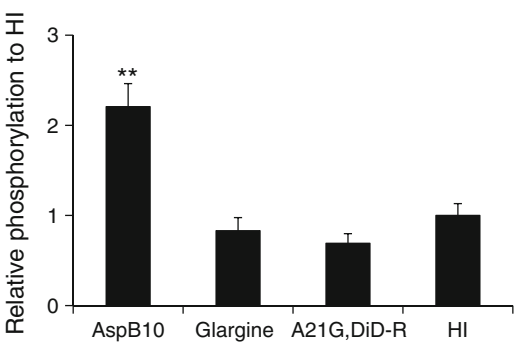

Fig. 5 Insulin glargine and non-metabolisable glargine did not increase Mvt-1 tumour growth. Mvt-1 tumour cells were injected at time 0 and growth was measured $(\mathbf{a}, \mathbf{b}) .12 .5 \mathrm{U} / \mathrm{kg}$ twice daily insulin glargine (white triangles), A21Gly,DiD-Arg (white diamonds) (a, b), AspB10 (white circles), or human insulin (black squares) (b) or vehicle treatment (black squares) (a), began as indicated by the vertical arrow ( $n=8-9$ per group). Representative western blot (c) of tumour protein lysates comparing IGF1R/IR phosphorylation of Mvt-1 tumours after chronic treatment with

doses). There were no deaths during any of our studies. Therefore, this mouse model provides us with the unique opportunity to study the effects of chronic administration of high doses of insulin analogues and rhIGF-1 on breast cancer progression.

Our findings showing the lack of effect of insulin glargine on tumour progression in these models are consistent with the pre-marketing rodent insulin glargine carcinogenicity studies [23], and also with a prospective human study on insulin glargine demonstrating no increase in the incidence of breast cancers in new users of insulin glargine [3]. However, previous pre-clinical carcinogenicity studies and also the ORIGIN human study were designed to examine whether insulin glargine led to the development of spontaneous tumours, whereas we examined the progression of established cancers in our model. Our findings are consistent with a previous mouse study that examined the latency time for mammary tumour development in a p53 mutant mouse model. In that study, AspB10 insulin and IGF-1 significantly decreased tumour latency compared with vehicle, but tumor latency was comparable between NPH insulin, glargine and vehicle [24]. Insulin glargine has been shown in vitro to have growthpromoting effects on specific cancer cell lines that express high levels of the IGF-1R [6,9]. The lack of mitogenicity of insulin glargine in vivo, however, was believed to be due to the rapid metabolism of glargine to its M1 metabolite [7, 9,
12.5 U/kg twice daily AspB10 (AB10), insulin glargine (glargine), nonmetabolisable glargine (A21Gly,DiD-R) or human insulin (HI). MWM indicates the molecular mass marker lane (c). Densitometric analysis ( $n=9$ per group) revealed greater receptor phosphorylation in the AspB10-treated group than all other groups. ${ }^{* *} p<0.01$ for AspB10-treated group vs other treatment groups (d). Graphs represent the mean of each group and error bars represent SEM

24]. Even after administration of high doses of insulin glargine, we found that glargine was metabolised into the M1 metabolite and did not lead to tumour progression.

More surprising is our finding that the non-metabolisable glargine analogue A21Gly,DiD-Arg also did not promote tumour growth in vivo. Previous in vitro studies have found that A21Gly,DiD-Arg has similar affinity for IGF-1R and similar mitogenic potency as insulin glargine in SAOS-2 cells [8]. The A21Gly,DiD-Arg analogue allowed us to model the effects that insulin glargine may have in a tumour-bearing person with the postulated slow rate of metabolism of insulin glargine, and greater exposure to higher circulating levels of the parent glargine than the M1 metabolite. In SAOS-2 and MCF-7 cell lines, the mitogenic potency and affinity of both insulin glargine and A21Gly,DiD-Arg for IGF-1R is higher than that of human insulin and the M1 metabolite of glargine; nevertheless, insulin glargine and A21Gly,DiD-Arg have significantly lower mitogenic potency and affinity compared with IGF-1 [8]. However, the higher affinity of parental glargine insulin and non-metabolisable glargine insulin for IGF-1R in vitro does not translate into in vivo IGF-1R phosphorylation, nor into increased breast cancer growth, which is likely because in vivo systemic and local IGF-1 are present. These findings highlight the need for complementary in vitro and in vivo studies to examine the role of insulin, insulin analogues and IGFs in tumour progression. 
Overall, in view of the increased risk of breast cancer mortality in patients with type 2 diabetes, the findings of our study are reassuring in that at high doses, insulin analogues do not promote the progression of breast tumours.

Acknowledgements S961 was a gift from Novo Nordisk, Bagsværd, Denmark. BMS-536924 was a gift from Bristol-Myers Squib, Princeton, NJ, USA.

Funding Support for this study was provided by Sanofi to DLR. DLR also received research support from NIH/NCI 5R01CA128799, ADA 1-13-BS-108. EJG received funding from NIH/NCI 1K08CA190770.

Duality of interest UW and NT are employees of Sanofi, and DLR is a member of an Advisory Board for Sanofi. EJG, ATH and ZZ declare that there is no duality of interest associated with their contribution to the manuscript.

Contribution statement All authors contributed to the design of experiments, data interpretation, manuscript preparation, reviewing and editing and approval of the final version. ATH, ZZ and EJG conducted the in vivo studies. EJG and DLR are guarantors of this work.

\section{References}

1. Mann DM, Woodward M, Ye F, Krousel-Wood M, Muntner P (2009) Trends in medication use among US adults with diabetes mellitus: glycemic control at the expense of controlling cardiovascular risk factors. Arch Intern Med 169:1718-1720

2. Hemkens LG, Grouven U, Bender R et al (2009) Risk of malignancies in patients with diabetes treated with human insulin or insulin analogues: a cohort study. Diabetologia 52:1732-1744

3. ORIGIN Trial Investigators, Gerstein HC, Bosch J et al (2012) Basal insulin and cardiovascular and other outcomes in dysglycemia. N Eng1 J Med 367:319-328

4. Badrick E, Renehan AG (2014) Diabetes and cancer: 5 years into the recent controversy. Eur J Cancer 50:2119-2125

5. Boyle P (2009) A salutary tale-glargine insulin and cancer risk. Ecancermedicalscience 3:ed5

6. Kurtzhals P, Schaffer L, Sorensen A et al (2000) Correlations of receptor binding and metabolic and mitogenic potencies of insulin analogs designed for clinical use. Diabetes 49:999-1005

7. Lucidi P, Porcellati F, Candeloro P et al (2014) Glargine metabolism over $24 \mathrm{~h}$ following its subcutaneous injection in patients with type 2 diabetes mellitus: a dose-response study. Nutr Metab Cardiovasc Dis 24:709-716

8. Werner U, Korn M, Schmidt R, Wendrich TM, Tennagels N (2014) Metabolic effect and receptor signalling profile of a nonmetabolisable insulin glargine analogue. Arch Physiol Biochem $120: 158-165$
9. Sommerfeld MR, Muller G, Tschank G et al (2010) In vitro metabolic and mitogenic signaling of insulin glargine and its metabolites. PLoS One 5:e9540

10. Jonassen I, Havelund S, Hoeg-Jensen T, Steensgaard DB, Wahlund PO, Ribel U (2012) Design of the novel protraction mechanism of insulin degludec, an ultra-long-acting basal insulin. Pharm Res 29: 2104-2114

11. Ashwell SG, Home PD (2001) Insulin glargine: the first clinically useful extended-action insulin analogue. Expert Opin Pharmacother 2:1891-1902

12. Kurtzhals P (2007) Pharmacology of insulin detemir. Endocrinol Metab Clin North Am 36(Suppl 1):14-20

13. Fernandez AM, Kim JK, Yakar S et al (2001) Functional inactivation of the IGF-I and insulin receptors in skeletal muscle causes type 2 diabetes. Genes Dev 15:1926-1934

14. Novosyadlyy R, Lann DE, Vijayakumar A et al (2010) Insulinmediated acceleration of breast cancer development and progression in a nonobese model of type 2 diabetes. Cancer Res 70:741751

15. Pei XF, Noble MS, Davoli MA et al (2004) Explant-cell culture of primary mammary tumors from MMTV-c-Myc transgenic mice. In Vitro Cell Dev Biol Anim 40:14-21

16. Borowsky AD, Namba R, Young LJ et al (2005) Syngeneic mouse mammary carcinoma cell lines: two closely related cell lines with divergent metastatic behavior. Clin Exp Metastasis 22:47-59

17. Ferguson RD, Novosyadlyy R, Fierz Y et al (2012) Hyperinsulinemia enhances c-Myc-mediated mammary tumor development and advances metastatic progression to the lung in a mouse model of type 2 diabetes. Breast Cancer Res 14:R8

18. Gallagher EJ, Alikhani N, Tobin-Hess A et al (2013) Insulin receptor phosphorylation by endogenous insulin or the insulin analog AspB10 promotes mammary tumor growth independent of the IGF-I receptor. Diabetes 62:3553-3560

19. Bolli GB, Hahn AD, Schmidt R et al (2012) Plasma exposure to insulin glargine and its metabolites M1 and M2 after subcutaneous injection of therapeutic and supratherapeutic doses of glargine in subjects with type 1 diabetes. Diabetes Care 35:2626-2630

20. Fierz Y, Novosyadlyy R, Vijayakumar A, Yakar S, LeRoith D (2010) Insulin-sensitizing therapy attenuates type 2 diabetesmediated mammary tumor progression. Diabetes 59:686-693

21. Baricevic I, Jones DR, Roberts DL et al (2015) A framework for the in vitro evaluation of cancer-relevant molecular characteristics and mitogenic potency of insulin analogues. Carcinogenesis 36:1040 1050

22. Hansen BF, Glendorf T, Hegelund AC et al (2012) Molecular characterisation of long-acting insulin analogues in comparison with human insulin, IGF-1 and insulin X10. PLoS One 7:e34274

23. Stammberger I, Bube A, Durchfeld-Meyer B, Donaubauer H, Troschau G (2002) Evaluation of the carcinogenic potential of insulin glargine (LANTUS) in rats and mice. Int J Toxicol 21:171-179

24. ter Braak B, Siezen C, Speksnijder EN et al (2015) Mammary gland tumor promotion by chronic administration of IGF1 and the insulin analogue AspB10 in the p53R270H/(+)WAPCre mouse model. Breast Cancer Res 17:14 